\title{
Perineural invasion in prostate needle biopsy: Prognostic value on radical prostatectomy and active surveillance
}

\author{
Nuno Ramos, Alexandre Macedo, João Rosa, Miguel Carvalho \\ Urology Department, Garcia de Orta Hospital, Almada, Portugal.
}

\begin{abstract}
Summary Purpose: The aim of this study was to evaluate the clinical impact of perineural invasion (PNI) in prostate biopsy in patients submitted to radical prostatectomy and on active surveillance (AS).

Materials and methods: We performed a single center, retrospective, cohort study on patients diagnosed with clinically localized prostate cancer and submitted to radical prostatectomy between January 2010 and December 2016. We evaluated clinical and anatomopathological characteristics from the biopsy and radical prostatectomy specimen and correlated with biochemical recurrence (BCR) using a survival analysis. We also evaluated the impact of PNI in patients with criteria for active surveillance.

Results: The cohort analyzed consists of 107 patients, with a mean age of 63.1 years and a mean PSA prior to biopsy of 7.8 $\mathrm{ng} / \mathrm{ml}$. In prostate biopsy, $66.4 \%$ of the patients had a Gleason score of 6, 30.9\% had a Gleason score of 7, and $2.7 \%$ had a Gleason score of 8 or higher, with PNI being detected in 57 (53.3\%) of the patients. Regarding the anatomopathological characteristics of the surgical specimen, invasion of the seminal vesicles was observed in $6.5 \%$, lymph nodes involvement in 9.3\% and positive surgical margins in $27.1 \%$ of the cases. During follow-up, BCR was recorded in $24.3 \%$ of cases. Clinicopathological features were stratified according to the presence or absence of PNI, with statistical significance in relation to the Gleason Score $(p=0.001)$, pathologic T stage $(p=0.001), D^{\prime}$ Amico risk $(p=0.002)$ and upstaging of the Gleason score $(p=0.045)$. The survival analysis revealed a relationship between PNI and BCR (hazard ratio $=2.98 ; 95 \%$ CI: $1.36-6.58 ; p=0.007)$. Regarding the men potentially eligible for AS, the presence of PNI on the biopsy presented a significant relation with Gleason upgrade $(p=0.004)$ and extraprostatic extension $(p=0.017)$.

Conclusions: The presence of PNI in prostate biopsy is related to adverse anatomopathological factors, being a potential predictor of BCR and have a possible role in the selection of patients for AS.
\end{abstract}

KEY WORDS: Prostate cancer; Active Surveillance; Prostatectomy.

Submitted 22 July 2020; Accepted 3 September 2020

\section{INTRODUCTION}

Prostate cancer ( $\mathrm{PCa}$ ) is the second most commonly diagnosed cancer in men and the third cause of cancer related death $(1,2)$. The best treatment strategies in clinically localized PCa remains unclear and varies from defini- tive treatment options such as radical prostatectomy (RP), external beam radiotherapy and brachytherapy to conservative management strategies including active surveillance (AS) (2-5). Despite the use of adequate therapy in localized PCa, approximately $18 \%$ of patients will eventually experience biochemical recurrence $(\mathrm{BCR})(1,6)$. Pathological stage, preoperative prostate-specific antigen (PSA) levels and Gleason score (GS) are widely used as risk factors for BCR (7). In other hand, AS has been increasingly adopted to prevent overtreatment in men with low-risk prostate cancer (8). This strategy pretends to identify patients with clinically indolent tumors and avoid or delay definitive treatment, without compromising survival (9). Although the concept of AS is well established, there is no consensus regarding the optimal characteristics of patients who should be managed by this strategy. Therefore, there is a growing interest in the identification of new clinicopathological features in prostate needle biopsy specimens to improve the evaluation of the likelihood of BCR, as improving the selection of patients to AS $(2,4,10)$. In this setting, perineural invasion (PNI) has been increasingly recognized as prognostic marker (11). PNI is a histopathologic finding representing the infiltration of cancer cells in, through and/or around nerves and is present in 7\%-43\% of prostate needle biopsies with PCa $(4,12,13)$. PNI has been implicated in PCa cell proliferation and extraprostatic spread, and the presence of PNI has been shown to be associated with adverse oncological outcomes (1416). Despite of PNI being a potential determinant in $\mathrm{PCa}$ behaviour, the association between PNI and PCa progression is still a subject of debate. Due to the uncertainty regarding the role of PNI, we performed a study to evaluate the association of PNI in prostate needle biopsy with adverse pathological findings on RP specimens, as well the impact on BCR. We also pretend to evaluate the role of PNI in patients' selection for AS (11).

\section{MATERIALS AND METHOdS}

We performed a single center retrospective cohort study, on male patients, who underwent RP due to clinically localised PCa, from January 2010 to December 2016. All patients underwent a 12-core biopsy prior to RP and presence or absence of PNI was assessed. PNI was defined as the histopathologic finding of circumferential 
or longitudinal tracking of PCa cells along a nerve, within the perineural space. PNI was not always reported on pathology reports and we excluded patients with unknown PNI status to minimize the potential for misclassification bias. Clinical and the biopsy parameters were evaluated, including age, PSA level prior to surgery, prostate volume, number of positive cores, total percent of core involvement and GS. We also assessed histopathologic finding on RP specimens: GS, margin positivity, stage, seminal vesicle involvement and lymphatic invasion.

The primary objective of this study was to report the association between PNI in prostate needle biopsy and adverse pathological findings on RP specimen, specifically, the presence of extraprostatic extension, surgical Gleason upgrading, positive surgical margin, and lymph node involvement. Gleason upgrading was defined as pathologic GS higher than the GS in the prostate biopsy. A sub-analysis was focused on evaluating the role of PNI on $\mathrm{BCR}$ following RP, defined as two successive postoperative PSA values of $0.2 \mathrm{ng} / \mathrm{mL}$ or greater. A secondary objective of this study, was to evaluate the impact of PNI in prostate needle biopsy on the selection of patients for AS. The AS cohort was based on the patients who performed RP but could be potentially selected for AS, defined according to the Epstein criteria (clinical stage $\leq$ T2a, PSA density $<0.15 \mathrm{ng} / \mathrm{mL}, \mathrm{PSA}<10 \mathrm{ng} / \mathrm{mL}$, biopsy Gleason score $\leq 6, \leq 2$ positive biopsy cores, and $\leq 50 \%$ cancer involvement in any biopsy core). A descriptive analysis on the study population was performed. Categorical data were compared using Pearson's chisquared test and continuous variables with Student t-test. The biochemical recurrence-free survival was calculated through the Kaplan-Meier analysis. To estimate the prognostic value of PNI we used Cox proportional hazard regression. A two-sided $p$ value $<0.05$ was considered as statistically significant. Statistical analysis was performed using SPSS ${ }^{\circledR}$, version 23.0 (SPSS Inc., Chicago, IL, USA).

\section{RESULTS}

A total of 107 patients were included in the study, of whom 57 (53.3\%) had PNI of the biopsy specimens.

The demographic and the clinical characteristics of the patients are shown in Table 1. Patient age ranged from 48 to 73 years (mean, 63.1 years; standard deviation (SD), 5.3 years). Preoperative serum PSA levels ranged from 1.51 to $21.9 \mathrm{ng} / \mathrm{mL}$ (mean, $7.8 \mathrm{ng} / \mathrm{mL}$; SD, $3.9 \mathrm{ng} / \mathrm{mL}$ ) and the clinical T stage was Tlc in $48(44.9 \%)$ of the patients, T2a in 28 (26.2\%), T2b in 20 (18.7\%) and T2c in $11(10.3 \%)$. According to D'Amico risk classification $65(60,7 \%)$ patients were low risk, $29(27,1 \%)$ intermediate risk and $13(12,2 \%)$ high risk.

From the prostate biopsy specimens, 71 (66.4\%) had a Gleason score of 6, $33(30.9 \%)$ had a Gleason score of 7 , and $3(2.7 \%)$ had a Gleason score of 8 or higher. The mean number of cores involved by tumor was 4.83 (SD, $1.5)$, and the mean percent of core involvement was $25.8 \%(S D, 20 \%)$.

Pathologic findings at RP are summarized in Table 2. Regarding the RP specimens, 25 (23.4\%) showed extraprostatic extension and 29 (27.1\%) had positive
Table 1.

Demographic, clinical and prostate biopsy characterization.

\begin{tabular}{|c|c|c|c|c|}
\hline & $\begin{array}{c}\text { PNI absence } \\
(n=50,46.7 \%)\end{array}$ & $\begin{array}{c}\text { PNI presence } \\
(n=57,53.3 \%)\end{array}$ & $\begin{array}{c}\text { Total } \\
(\mathrm{n}=107)\end{array}$ & p \\
\hline Age, mean (years) & 62.4 (SD 5.2) & 63.7 (SD 5.4) & 63.1 (SD 5.38) & 0.189 \\
\hline PSA level $(\mathrm{ng} / \mathrm{ml})$ & 7.64 (SD 3.6) & 7.94 (SD 4.5) & 7.8 (SD 3.9) & 0.53 \\
\hline Prostate volume(mean, gr) & 46.5 (SD 17.5) & 45.1 (SD 14.8) & 45.8 (SD 16.1) & 0.46 \\
\hline GS biopsy & & & & 0.036 \\
\hline 6 & $39(78 \%)$ & $32(56.1 \%)$ & $71(66.4 \%)$ & \\
\hline 7 & $10(20 \%)$ & $23(40.3 \%)$ & $33(30.9 \%)$ & \\
\hline$\geq 8$ & $1(2 \%)$ & $2(3.6 \%)$ & $3(2.7 \%)$ & \\
\hline Number of positive cores (mean) & 4.56 (SD 2.36) & 5.07 (SD 2.77) & 4.83 (SD 2.5) & 0.31 \\
\hline Percent of core involvement (mean) & $19.48 \%$ (SD 17\%) & $31.3 \%($ SD 20.95\%) & $25.8 \%$ (SD 20\%) & 0.04 \\
\hline Clinical stage & & & & 0.019 \\
\hline T1c & $23(46 \%)$ & $25(43.9 \%)$ & $48(44.9 \%)$ & \\
\hline T2a & $20(40 \%)$ & $8(14 \%)$ & $28(26.2 \%)$ & \\
\hline $\mathrm{T} 2 \mathrm{~b}$ & $6(12 \%)$ & $14(24.6 \%)$ & $20(18.7 \%)$ & \\
\hline $\mathrm{T} 2 \mathrm{C}$ & $1(2 \%)$ & $10(17.6 \%)$ & $11(10.3 \%)$ & \\
\hline
\end{tabular}

Table 2.

Clinical and histopathologic finding on RP specimens' characterization.

\begin{tabular}{|c|c|c|c|c|}
\hline & $\begin{array}{c}\text { PNI absence } \\
(n=50,46.7 \%)\end{array}$ & $\begin{array}{c}\text { PNI presence } \\
(n=57,53.3 \%)\end{array}$ & $\begin{array}{c}\text { Total } \\
(n=107)\end{array}$ & $p$ \\
\hline$\overline{D^{\prime} A m i c o ~ r i s k ~}$ & & & & 0.002 \\
\hline Low & $38(76 \%)$ & $27(47.4 \%)$ & $65(60.7 \%)$ & \\
\hline Intermediate & $10(20 \%)$ & $19(33.3 \%)$ & $29(27.1 \%)$ & \\
\hline High & $2(4 \%)$ & $11(19.3 \%)$ & $13(12.2 \%)$ & \\
\hline Gleason score & & & & 0.001 \\
\hline 6 & $34(68 \%)$ & $20(35.1 \%)$ & $71(66.4 \%)$ & \\
\hline 7 & $16(32 \%)$ & $34(59.6 \%)$ & $33(30.9 \%)$ & \\
\hline$\geq 8$ & - & $3(5.3 \%)$ & $3(2.7 \%)$ & \\
\hline Pathological stage & & & & 0.001 \\
\hline pT2a & $8(16 \%)$ & $7(12.3 \%)$ & $15(14 \%)$ & \\
\hline pT2b & $5(10 \%)$ & $1(1.8 \%)$ & $6(5.6 \%)$ & \\
\hline $\mathrm{pT} 2 \mathrm{c}$ & $33(66 \%)$ & $27(47.4 \%)$ & $60(56.1 \%)$ & \\
\hline$\geq \mathrm{pT} 3$ & $4(8 \%)$ & $22(38.6 \%)$ & $26(24.3 \%)$ & \\
\hline GS upgrading & $8(16 \%)$ & $18(31.6 \%)$ & $26(24.2 \%)$ & 0.065 \\
\hline Lymphatic invasion & $3(6 \%)$ & $7(12.3 \%)$ & $10(9.3 \%)$ & 0.27 \\
\hline SV invasion & $1(2 \%)$ & $6(10.5 \%)$ & $7(6.5 \%)$ & 0.11 \\
\hline Extraprostatic extension & $4(8 \%)$ & $21(36.8 \%)$ & $25(23.4 \%)$ & 0.001 \\
\hline Margin positivity & $8(16 \%)$ & $17(29.8 \%)$ & $25(23.4 \%)$ & 0.09 \\
\hline BCR & $9(18 \%)$ & $22(38.6 \%)$ & $31(29 \%)$ & 0.032 \\
\hline \multicolumn{5}{|c|}{$\begin{array}{l}\text { Clinical and histopathologic finding on RP specimens stratified in two groups: patients with PNI absence and patients } \\
\text { with PNI presence. Categorical data were compared using Pearson's chi-squared test and continuous variables with } \\
\text { Student t-test. SV-seminal vesicle. }\end{array}$} \\
\hline
\end{tabular}

margins. The seminal vesicles were invaded in 7 (6.5\%) cases and lymph nodes were involved in 10 (9.3\%). Surgical GS upgrading was observed in 26 (24.2\%) patients. The mean follow-up time was 71 months (SD 25.3 months) and, in this period, 26 (24.3\%) men experienced $\mathrm{BCR}$.

Clinical and anatomopathological characteristics from the biopsy and RP specimen were stratified according to the presence or absence of PNI. Significant differences were found, patients with PNI presented higher GS on biopsy and RP specimen $(\mathrm{p}=0.036$ and $\mathrm{p}=0.001)$, 
Figure 1.

Kaplan-Meier curve and Log rank test.

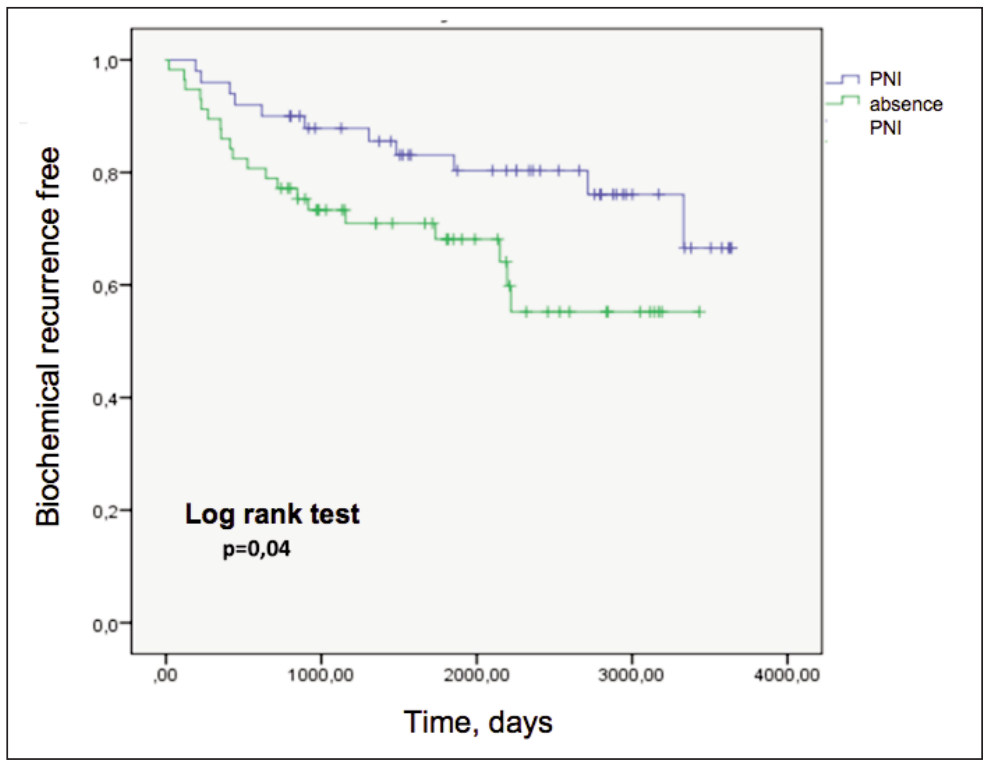

Biochemical recurrence-free survival in patients with or without PNI.

Table 3.

Association between disease characteristics and biochemical recurrence-free survival.

\begin{tabular}{|c|c|c|c|c|c|c|}
\hline & \multicolumn{3}{|c|}{ Univariate } & \multicolumn{3}{|c|}{ Multivariate } \\
\hline & HR & $(95 \%$ IC) & $P$ value & HR & (95\% IC) & $P$ value \\
\hline \multicolumn{7}{|c|}{ GS in RP specimen } \\
\hline$<7$ & 1 & & & & & \\
\hline$\geq 7$ & 3.01 & $(1.34-6.76)$ & 0.008 & 1.65 & $(1.04-2.60)$ & 0.032 \\
\hline \multicolumn{7}{|c|}{ Extraprostatic extension } \\
\hline absence & 1 & & & & & \\
\hline presence & 4.02 & $(1.98-8.15)$ & 0.0001 & 1.26 & $(0.48-3.27)$ & 0.64 \\
\hline \multicolumn{7}{|c|}{ Surgical margin } \\
\hline negative & 1 & & & & & \\
\hline positive & 3.89 & $(1.90-7.94)$ & 0.0001 & 3.51 & $(1.45-8.52)$ & 0.005 \\
\hline \multicolumn{7}{|l|}{$\overline{\mathrm{PN}}$} \\
\hline absence & 1 & & & & & \\
\hline presence & 2.98 & $(1.36-6.58)$ & 0.007 & 1.64 & $(0.7-3.86)$ & 0.25 \\
\hline
\end{tabular}

Table 4.

Clinical and histopathologic finding on active surveillance patients.

\begin{tabular}{|lcccc|}
\hline & $\begin{array}{c}\text { PNI absence } \\
\text { (n= 16, 55.2\%) }\end{array}$ & $\begin{array}{c}\text { PNI presence } \\
\text { (n= 13, 44.8\%) }\end{array}$ & $\begin{array}{c}\text { Total } \\
\text { (n= 29) }\end{array}$ & p \\
\hline Age, mean (years) & $62.5($ SD 4.9) & $63.3($ SD 6.7) & $62.86(52-73)$ & 0.362 \\
\hline PSA level (ng/ml) & $7.31($ SD 4.9) & $7($ SD 4.1) & $7.1($ (SD 4.2) & 0.41 \\
\hline Prostate volume (mean, gr) & $56.9($ SD 24.5) & $48.7($ (SD 18.4) & $53.3($ SD 22) & 0.31 \\
\hline GS upgrading & $1(6.3 \%)$ & $7(53.8 \%)$ & $8(27.6 \%)$ & 0.004 \\
\hline extraprostatic extension & - & $4(30.8 \%)$ & $4(13.8 \%)$ & 0.017 \\
\hline $\begin{array}{l}\text { CSub-group of patients potentially selected to active surveillance. Clinical and histopathologic finding stratified in two } \\
\text { groups: patients with PNI absence and patients with PNI presence in the prostate biopsy. }\end{array}$ & & \\
\hline
\end{tabular}

higher percent of core involvement $(p=0.04)$, higher clinical and pathologic $T$ stage $(p=0.019$ and $p=0.001)$, higher D'Amico risk $(p=0.002)$, extraprostatic extension $(p=0.001)$, and higher BCR $(p=0.032)$ comparing with patients without PNI. On other hand, no significant differences were identified with respect to age $(p=0.189)$, preoperative PSA $(\mathrm{p}=0.53)$, number of positive cores $(\mathrm{p}=0.31)$, GS upgrading $(\mathrm{p}=0.065)$, surgical margin involvement $(p=0.09)$, lymph node invasion $(p=0.27)$ and seminal vesicles involvement $(\mathrm{p}=0.11)$.

The Kaplan-Meier curve (Figure 1) revealed a poorer recurrence-free survival in patients with PNI (Log rank test $p=0.04$ ). On univariate Cox analysis (Table 3), PNI was associated with BCR (HR: 2.98, 95\% CI: 1.36$6.58, p=0.007$ ), as GS on RP specimen (HR: 3.01, 95\% CI: 1.34-6.76, $\mathrm{p}=0.008)$, surgical margin positivity (HR: 3.86, 95\% CI: 1.907.94, $\mathrm{p}=0.0001$ ) and presence of extraprostatic extension (HR: 4.02, 95\% CI: 1.98-8.15 $\mathrm{p}=0.0001)$. However, the prognostic role of PNI disappeared in multivariate analysis when adjusted for other predictive factors. In the cohort, a total of 29 men submitted to $\mathrm{RP}$ were potentially eligible for AS, of whom 13 (44.8\%) had biopsy PNI. The characteristics of this subgroup are listed in Table 4.

A significant relation between PNI and GS upgrade $(p=0.004)$ and extraprostatic extension $(p=0.017)$ was found in the AS group.

\section{Discussion}

The evaluation of pathological features that may predict oncologic outcomes are important for counselling patients and therapy selection, as treatment options in PCa differ according to defined risk groups (17). PNI was identified as a possible significant marker of adverse pathologic findings in localized PCa, however, the oncological significance and prognostic value is still controversial, with inconsistent results among studies (13).

The incidence of PNI in biopsy specimens in the present study is similar to that reported by Ravery et al. (53,3\% VS $47 \%)(18,19)$. Although, a wide range of incidences have been reported in the literature due to variation in pathologic definitions and interpretation of PNI (16). There are many challenges in looking for PNI and a negative result may either indicate that there no nerves identified in the biopsy or that nerves were present without invasion (2).

On our study, PNI presence on the biopsy cores was associated with adverse parameters in RP specimens, including higher GS, higher pathologic $\mathrm{T}$ staging and extraprostatic extension. Such associations were also reported in a recent meta-analysis were PNI was a significant marker to predict high stage disease (4).

Despite hypothesized that spread along intraprostatic nerves may facilitating extraprostatic tumoral extension, there are controversial results in studies investigating the correlation between PNI and BCR. Jeon et al. and Kang et al. showed that PNI is associated with adverse pathologic findings and is an independent predictor for BCR in PCa patients who undergo $\mathrm{RP}(6,20)$. The same results were observed by Yu et al. and Wong et al. in patients who undergo external beam radiotherapy $(21,22)$. On the 
contrary, Reeves et al. and Freedland et al. reported that PNI is not correlated with BCR in PCa after RP $(23,24)$. In our cohort, we found an association between PNI and BCR on univariate but not on multivariate analysis. This questions whether PNI is an independent prognostic factor or just a risk factor for BCR, since significance is lost when PNI presence is controlled for other biopsy parameters, such as Gleason score and extraprostatic extension (25). One possible explanation for these findings is that PNI may only be an important prognostic factor in a specific sub-group of patients. D'Amico et al. found that biopsy PNI showed statistical significance on multivariate analysis only in the low risk group and Quinn et al. reported that biopsy PNI was a significant prognostic factor on multivariate analysis of patients with PSA values more than $10 \mathrm{ng} / \mathrm{ml}(26,27)$.

In the same setting, rather than evaluated the presence or absence of PNI, quantification could have a better predictive value. Maru et al. found that PNI diameter $>0.25$ $\mathrm{mm}$ was an independent prognostic indicator for biochemical recurrence on multivariate analysis (28). Moreover, Sun et al. demonstrated that multifocal PNI, rather than unifocal PNI, is correlate to shorter biochemical recurrence-free survival in patients with PCa (10).

AS has been widely accepted as an observational strategy, in the last decade, in response to the over-treatment of men with low-risk PCa (16). The selection of patients is based on pathologic findings on needle core biopsy. At present, biopsy PNI is not included in the established criteria for AS selection, thus, whether PNI is potentially associated with worse prognosis and preclude a conservative management is not known. In our analysis, the presence of PNI in patients who met criteria for AS has been associated with adverse pathologic findings at prostatectomy, including GS upstaging and extraprostatic extension. These finding suggest a potential role for biopsy PNI in identifying men at risk for progression on AS. This evidence is corroborated by a retrospective review of the REduction by Dutasteride of clinical progression Events in Expectant Management (REEDEM) study, with 302 men on AS, who describe that PNI is an independent predictor of clinical progression (73\% after 2 years) (29). Similarly, in a cohort of 165 men on AS, Cohn et al. reported that biopsy PNI remained a significant predictor for AS failure after adjustment biopsy parameters such as tumour length (30). While these data suggest that patients with biopsy PNI on initial biopsy may not be good candidates for AS, future study is required to assess prognostic value of this pathologic finding.

Several limitations should be acknowledged in this study. First, the small number of cases, similar to other series published, limits the statistical power of the conclusions. Second, the study design, as a single-institution retrospective analysis with risk of unmeasured bias, does not allow to generalize the conclusions. Third, prostatectomy specimens did not undergo a centralized review, so there may be variability in the reporting of PNI. Ideally, a multicenter randomized prospective study with a larger sample would answer many questions raised in our study. Fourth, we only report PNI as a binary variable because quantitative measures of PNI were not included in pathology reports. Finally, a short follow-up with a mean of 71 months can undervalue the BCR.

\section{Conclusions}

In conclusion, despite the limitations listed above, this study recognizes the clinicopathological importance and potential prognostic value of PNI in PCa. The presence of PNI on prostate biopsy cores is an important predictive of aggressive disease in patients submitted to RP with clinically localized PCa and an indicator of BCR in univariate analysis. Additionally, among men who met criteria for AS, biopsy PNI is associate with GS upgrade and extraprostatic extension and could have a role in the selection of patients for AS. A large prospective study with longer follow-up is needed to confirm these results.

\section{REFERENCES}

1. Zhang $L J$, Wu B, Zha ZL, et al. Perineural invasion as an independent predictor of biochemical recurrence in prostate cancer following radical prostatectomy or radiotherapy: a systematic review and meta-analysis. BMC Urol. 2018; 18:5.

2. Ahmad AS, Parameshwaran V, Beltran L, et al. Should reporting of peri-neural invasion and extra prostatic extension be mandatory in prostate cancer biopsies? correlation with outcome in biopsy cases treated conservatively. Oncotarget. 2018; 9:20555-20562.

3. Vargas SO, Jiroutek M, Welch WR, et al. Perineural invasion in prostate needle biopsy specimens. Correlation with extraprostatic extension at resection. Am J Clin Pathol. 1999; 111:223-228.

4. Celik S, Bozkurt O, Demir O, et al. Effects of perineural invasion in prostate needle biopsy on tumor grade and biochemical recurrence rates after radical prostatectomy. Kaohsiung J Med Sci. 2018; 34:385-390.

5. Erdem S, Verep S, Bagbudar S, et al. The clinical predictive factors and postoperative histopathological parameters associated with upgrading after radical prostatectomy: A contemporary analysis with grade groups. Prostate. 2020; 80:225-234.

6. Jeon HG, Bae J, Yi JS, et al. Perineural invasion is a prognostic factor for biochemical failure after radical prostatectomy. Int J Urol. 2009; 16:682-686.

7. Barsky AR, Kraus RD, Carmona R, et al. Investigating association of perineural invasion on prostate biopsy with Gleason score upgrading at prostatectomy: A multi-institutional analysis. Cancer Med. 2020; 9:3383-3389.

8. Verep S, Erdem S, Ozluk Y, et al. The pathological upgrading after radical prostatectomy in low-risk prostate cancer patients who are eligible for active surveillance: How safe is it to depend on bioptic pathology? Prostate. 2019; 79:1523-1529.

9. Ediz C, Akan S, Temel MC, Yilmaz O. The importance of PSADensity in active surveillance for prostate cancer. Arch Ital Urol Androl. 2020; 92:136-141.

10. Kraus RD, Barsky A, Ji L, et al. The Perineural Invasion Paradox: Is Perineural Invasion an Independent Prognostic Indicator of Biochemical Recurrence Risk in Patients With pT2NORO Prostate Cancer? A Multi-Institutional Study. Adv Radiat Oncol. 2019; 4:96-102

11. Turner RM, $2^{\text {nd }}$, Yecies TS, Yabes JG, et al. Biopsy Perineural Invasion in Prostate Cancer Patients Who Are Candidates for Active Surveillance by Strict and Expanded Criteria. Urology. 2017; 102:173-177.

12. Strom P, Nordstrom T, Delahunt B, et al. Prognostic value of perineural invasion in prostate needle biopsies: a population-based study of patients treated by radical prostatectomy. J Clin Pathol. 2020. 
13. Zhao J, Chen J, Zhang $M$, et al. The clinical significance of perineural invasion in patients with de novo metastatic prostate cancer. Andrology. 2019; 7:184-192.

14. Zareba P, Flavin R, Isikbay $M$, et al. Perineural Invasion and Risk of Lethal Prostate Cancer. Cancer Epidemiol Biomarkers Prev. 2017; 26:719-726.

15. Kuang AG, Nickel JC, Andriole GL, et al. Both acute and chronic inflammation are associated with less perineural invasion in men with prostate cancer on repeat biopsy. BJU Int. 2019; 123:91-97.

16. Wu S, Lin X, Lin SX, et al. Impact of biopsy perineural invasion on the outcomes of patients who underwent radical prostatectomy: a systematic review and meta-analysis. Scand J Urol. 2019; 53:287-294.

17. Camur E, Coskun A, Kavukoglu, et al. Prostate volume effect on Gleason score upgrading in active surveillance appropriate patients. Arch Ital Urol Androl. 2019; 91:93-96.

18. Ravery V, Boccon-Gibod LA, Dauge-Geffroy MC, et al. Systematic biopsies accurately predict extracapsular extension of prostate cancer and persistent/recurrent detectable PSA after radical prostatectomy. Urology. 1994; 44:371-376.

19. Peng LC, Narang AK, Gergis C, et al. Effects of perineural invasion on biochemical recurrence and prostate cancer-specific survival in patients treated with definitive external beam radiotherapy. Urol Oncol. 2018; 36:309 e307-309 e314.

20. Kang $M$, Oh JJ, Lee $S$, et al. Perineural Invasion and Lymphovascular Invasion are Associated with Increased Risk of Biochemical Recurrence in Patients Undergoing Radical Prostatectomy. Ann Surg Oncol. 2016; 23:2699-2706.

21. Wong WW, Schild SE, Vora SA, Halyard MY. Association of percent positive prostate biopsies and perineural invasion with biochemical outcome after external beam radiotherapy for localized prostate cancer. Int J Radiat Oncol Biol Phys. 2004; 60:24-29.

22. Yu HH, Song DY, Tsai YY, et al. Perineural invasion affects bio- chemical recurrence-free survival in patients with prostate cancer treated with definitive external beam radiotherapy. Urology. 2007; 70:111-116.

23. Reeves F, Hovens CM, Harewood L, et al. Does perineural invasion in a radical prostatectomy specimen predict biochemical recurrence in men with prostate cancer? Can Urol Assoc J. 2015; 9:E252255.

24. Freedland SJ, Csathy GS, Dorey F, Aronson WJ. Percent prostate needle biopsy tissue with cancer is more predictive of biochemical failure or adverse pathology after radical prostatectomy than prostate specific antigen or Gleason score. J Urol. 2002; 167:516-520.

25. Trpkov C, Yilmaz A, Trpkov K. Perineural invasion in prostate cancer patients who are potential candidates for active surveillance: validation study. Urology. 2014; 84:149-152.

26. D'Amico AV, Wu Y, Chen MH, et al. Perineural invasion as a predictor of biochemical outcome following radical prostatectomy for select men with clinically localized prostate cancer. J Urol. 2001; 165:126-129.

27. Quinn DI, Henshall SM, Brenner PC, et al. Prognostic significance of preoperative factors in localized prostate carcinoma treated with radical prostatectomy: importance of percentage of biopsies that contain tumor and the presence of biopsy perineural invasion. Cancer. 2003; 97:1884-1893.

28. Maru N, Ohori M, Kattan MW, et al. Prognostic significance of the diameter of perineural invasion in radical prostatectomy specimens. Hum Pathol. 2001; 32:828-833.

29. Moreira DM, Fleshner NE, Freedland SJ. Baseline Perineural Invasion is Associated with Shorter Time to Progression in Men with Prostate Cancer Undergoing Active Surveillance: Results from the REDEEM Study. J Urol. 2015; 194:1258-1263.

30. Cohn JA, Dangle PP, Wang CE, et al. The prognostic significance of perineural invasion and race in men considering active surveillance. BJU Int. 2014; 114:75-80.

\section{Correspondence}

Nuno Ramos, MD (Corresponding Author)

nuno.ramos@hgo.min-saude.pt

nunoandre33@gmail.com

Alexandre Macedo, MD

alex.m.macedo89@gmail.com

João Rosa, MD

jpmrosa@yahoo.com

Miguel Carvalho, MD

uro.miguelcarvalho@gmail.com

Urology Department, Garcia de Orta Hospital

Av. Torrado da Silva, 2801-951, Almada (Portugal) 